\title{
Development of a Reference Material for Image Sharpness Evaluation in Scanning Electron Microscopy
}

\author{
Kazuhiro Kumahai ${ }^{1}$ and Akira Kurokawa ${ }^{1}$
}

1. Surface and Nano Analysis Research Group, Research Institute for Material and Chemical Measurement, National Metrology Institute of Japan, National Institute of Advanced Industrial Science and Technology (AIST), Tsukuba, Japan

The spatial resolution of micrographs of scanning electron microscopy (SEM) is one of biggest interests for both of instrument developers and users, since the resolution is one of the indicators to measure the performance of SEM instruments. At present, in the typical practice of specifying the resolution of SEM images, of measuring the smallest visible gap between two particles in an image, there still is poor repeatability and ambiguity owing to the selecting of the smallest gap by human operator. Thus, the international standards organization technical committee on microbeam analysis, ISO/TC202, is developing a standard method for the quantitative determination of image sharpness in SEM images applying derivative (DR) method [1,2].

On the other hand, because of the material dependent nature of SEM image, there are needs for standard specimen for actual use of the image sharpness evaluation, for instance, check up of instrument status and inter-instrument comparison. To meet these demands, the authors are developing a reference material designed for image sharpness evaluation. In this paper, we report the recent progress on a prototype of the reference material.

According to the DR method procedure [1], specimen for image sharpness determination should (1) consists of particles on background (2) show enough contrast between the particles and the background (3) has no contrast anomalies such as charging effect or strong edge effect. To meet these requirements, we designed the prototype and fabricated one by a semiconductor process. Figure 1 illustrates the structure of the prototype. Our prototype consists of arrays of W cylinder embedded in Si substrate, which looks dot array when observed from surface normal. The diameter of $\mathrm{W}$ dots has fourteen degrees from $60 \mathrm{~nm}$ to $125 \mathrm{~nm}$, enabling us to choose the optimum size of dot according to observing magnification. The depth of cylinder is designed to be $100 \mathrm{~nm}$. To increase usability, the period $d$ between two neighboring dots is known. This can be used to calibrate SEM to determine the pixel size of an image, prior to the determination of image sharpness.

As shown Fig. 2, the tungsten dots in SE image have bright contrast against silicon background that is large enough for the image sharpness determination. This bright contrast is stable over accelerating voltage range of conventional SEM. Line-profiles extracted from the edge of $\mathrm{W}$ dot have small fluctuation, shown in Fig 3, suggesting that the interface between W cylinder and the substrate has high uniformity.

To elucidate the quality and the limitation of the prototype, we observed cross-section of tungsten cylinders by using SEM and transmission electron microscopy. Surface morphology of the tungsten dots is also evaluated with atomic force microscopy. In the presentation, the details of these analyses of the prototype, and the result of image sharpness determination under various conditions will be discussed to improve the prototype toward more sophisticated reference material. 


\section{References:}

[1] ISO/TS 24597:2011 Microbeam analysis — Scanning electron microscopy — Methods of evaluating image sharpness (2011)

[2] M Matthews and J Shah, Microsc. Microanal. 21 (2015) p. 2239.

[3] A part of this study was conducted as one of the activities in Japan Research Industries and Industrial Technology Association supported by JKA Foundation (FY2015).

(a)

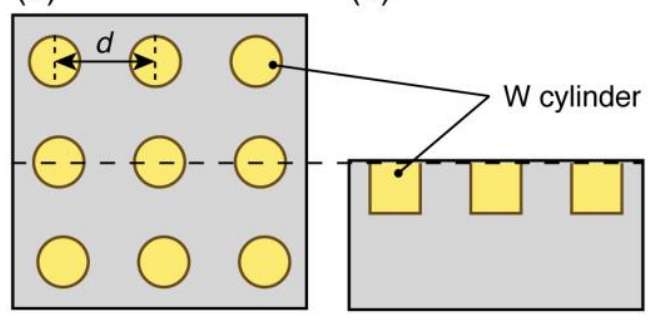

Figure 1. Schematics of the prototype of the reference material, which consists of $\mathrm{W}$ dot array on $\mathrm{Si}$ substrate. (a) Top view and (b) side view. The period $d$ between two neighboring dots is known.
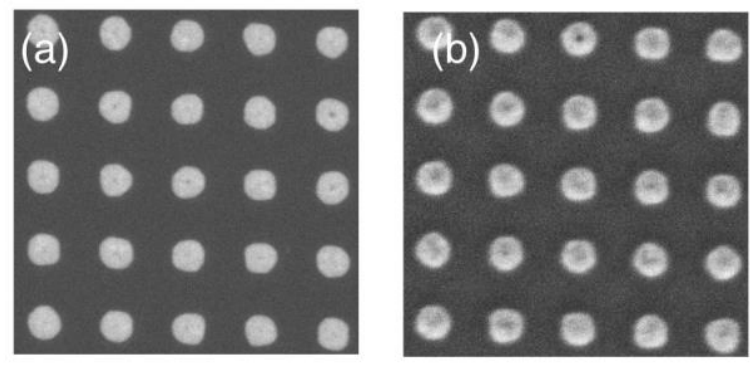

$200 \mathrm{~nm}$

Figure 2. Typical SE images of the prototype taken by FE-SEM at accelerating voltages of (a) $15 \mathrm{kV}$, and (b) $3 \mathrm{kV}$.

(a)

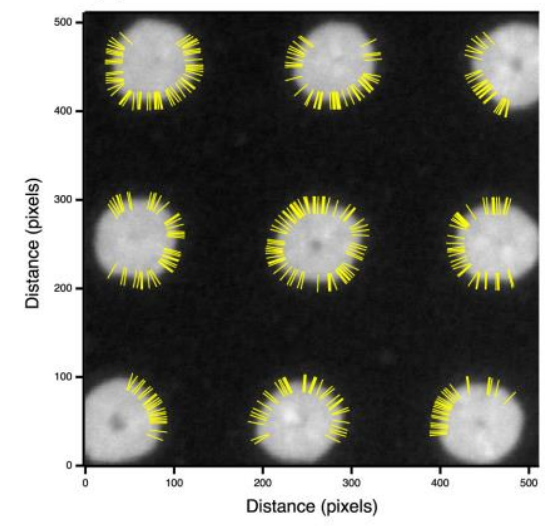

(b)

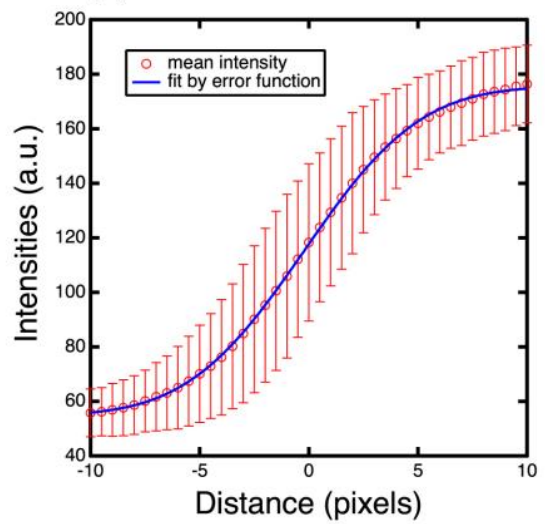

Figure 3. (a) An example of line-profile extraction on the particle edge for image sharpness determination. Yellow lines show the position of extracted line-profiles. (b) Mean grey-scale value of the extracted 348 line-profiles in (a). Error bars represent the 95\% confidence interval ( 2 standard deviations). 